\title{
SEMILOCAL GROUP RINGS IN CHARACTERISTIC ZERO
}

\author{
JOHN LAWRENCE AND ȘHEILA M. WOODS ${ }^{1}$
}

\begin{abstract}
It is shown that if $F$ is a field of characteristic zero and $G$ is a group such that the group ring $F[G]$ is semilocal then $G$ must be finite. A generalization to group rings over rings is given.
\end{abstract}

A ring $R$ is semilocal if $R / J(R)$ is artinian, where $J(R)$ denotes the Jacobson radical of $R$. $R$ is said to be local if $R / J(R)$ is a division ring. It is well known that the group ring is never local for a field of characteristic zero unless the group is trivial. As it is conjectured that $J(F[G])=(0)$ whenever $F$ is a field of characteristic zero, we expect $F[G]$ semilocal to imply $G$ finite, and this is easily proved if $F$ is not algebraic over the rationals, by a theorem of Amitsur [1]. The result in this paper may be interpreted as saying that the radical of the rational group ring cannot be "too large".

We would like to thank D. S. Passman for his helpful suggestions.

LEMMA 1. Let $K$ be a central subfield of a division ring $D$ and let $T \in M_{n}(D)$, the full $n$ by $n$ matrix ring over $D$. Then the set $S(T)=\left\{k \in K: 1-k^{-1} T\right.$ is singular\} has at most $n$ elements.

Proof. When written on the right, the elements of $M_{n}(D)$ may be regarded as left $D$-linear transformations from the vector space $D^{n}$ to $D^{n}$. If $1-k^{-1} T$ is singular, there is a nonzero vector $v \in D^{n}$ such that $v\left(1-k^{-1} T\right)=0$, or $v T=k v$ since $k$ commutes with $v$. Hence $k$ is an eigenvalue of $T$. Standard arguments of linear algebra show that eigenvectors in $D^{n}$ corresponding to distinct eigenvalues of $T$ in the centre of $D$ are $D$-linearly independent.

Corollary. Let $R$ be a completely reducible $K$-algebra and let $x \in R$. Then the set $S(x)=\left\{k \in K: 1-k^{-1} x\right.$ is not a unit in $\left.R\right\}$ is finite.

The following clever lemma forms a major part of the proof of the theorem in [3].

LEMMA 2 (FORMANEK). Let $K$ be a subfield of the reals and let $x=\sum_{i=1}^{n} a_{i} g_{i}$

Received by the editors February 23, 1976.

AMS (MOS) subject classifications (1970). Primary 16A26, 16A46; Secondary 16A10.

Key words and phrases. Group ring, semilocal ring.

1 The research of both authors was supported by the National Research Council of Canada. It was carried out while the second author was visiting the University of Wisconsin-Madison.

Copyright $\odot$ 1977, American Mathematical Society 
$\in K[G]$ be such that each $a_{i}>0$ and $\sum_{i=1}^{n} a_{i}<1$. If $1-x$ is a unit in $K[G]$ then the group generated by $\left\{g_{1}, g_{2}, \ldots, g_{n}\right\}$ is finite.

Proof. It is sufficient to show that the semigroup $H$ generated by $\left\{g_{1}, g_{2}, \ldots, g_{n}\right\}$ is finite, since a finite semigroup with cancellation is a group. This is done by showing that $H$ is contained in the support of $(1-x)^{-1}$.

The norm || defined on $K[G]$ by $\left|\sum k_{i} g_{i}\right|=\sum\left|k_{i}\right|$ satisfies $|y|>0$ for all $y \neq 0$ and $|y z| \leqslant|y||z|$ for all $y, z \in K[G]$.

Let $(1-x)^{-1}=y$ and for $m \geqslant 0$ let $y_{m}=1+x+\cdots+x^{m}$. Then $y-y_{m}$ $=y(1-x)\left(y-y_{m}\right)=y\left[1-\left(1-x^{m+1}\right)\right]=y x^{m+1}$ and

$$
\left|y-y_{m}\right| \leqslant|y||x|^{m+1}=|y|\left(\sum_{i=1}^{n} a_{i}\right)^{m+1} .
$$

Hence $\lim _{m \rightarrow \infty}\left|y-y_{m}\right|=0$.

Let $h \in H$. Then $h=g_{i_{1}} g_{i_{2}} \cdots g_{i_{r}}$ for some $r>0$. Since all the coefficients in $x$ are positive, there can be no cancellation of terms in the powers of $x$; hence $h \in \operatorname{Supp}\left(x^{r}\right)$. Moreover for all $m \geqslant r, h \in \operatorname{Supp}\left(y_{m}\right)$ and the coefficient of $h$ in $y_{m}$ is at least $a_{i_{1}} a_{i_{2}} \cdots a_{i_{r}}$. If $h \notin \operatorname{Supp}(y)$ this implies that $\left|y-y_{m}\right| \geqslant a_{i_{1}} a_{i_{2}} \cdots a_{i_{r}}$, contradicting $\lim _{m \rightarrow \infty}\left|y-y_{m}\right|=0$.

THEOREM. Let $F$ be a field of characteristic zero and let $G$ be a group. If the group ring $F[G]$ is semilocal then $G$ is finite.

Proof. We first prove that $G$ is locally finite. Let $\left\{g_{1}, g_{2}, \ldots, g_{n}\right\}$ be a finite subset of $G$ and let $x=g_{1}+g_{2}+\cdots+g_{n}$. As $F[G]$ is semilocal, $\overline{F[G]}$ $=F[G] / J(F[G])$ is completely reducible. Hence by the Corollary to Lemma 1 there exists an integer $m>n$ such that $\overline{1-m^{-1} x}$ is a unit in $\overline{F[G]}$. Thus $1-m^{-1} x$ is a unit in $F[G]$. By considering a $Q$-basis for $F$ (where $Q$ denotes the rationals) we see that $1-m^{-1} x$ is a unit in $Q[G]$. By Lemma 2, the subgroup generated by $\left\{g_{1}, g_{2}, \ldots, g_{n}\right\}$ is finite. This proves that $G$ is locally finite.

By the Maschke theorem, $K[H]$ is completely reducible for every finitely generated subgroup $H$ of $G$. In particular, $J(K[H])=(0)$. Hence $J(K[G])$ $=(0)$ and $K[G]$ is completely reducible. It follows, again from the Maschke theorem, that $G$ is finite. (For more details, see [2].)

COROLlary. Let $A$ be a ring such that $A / J(A)$ has characteristic zero and let $G$ be a group. Then the group ring $A[G]$ is semilocal if and only if $A$ is semilocal and $G$ is finite.

Proof. Suppose $A$ is semilocal and $G$ is finite. By [2, Proposition 9], $J(A) A[G] \subseteq J(A[G])$. It follows that $A[G] / J(A[G])$ is a homomorphic image of $A[G] /(J(A) A[G]) \cong \bar{A}[G]$, an artinian ring (where $\bar{A}=A / J(A))$. Hence $A[G]$ is semilocal.

Conversely suppose $A[G]$ is semilocal. Since $A$ is a homomorphic image of $A[G], A$ is semilocal. Hence $\bar{A} \cong \bigoplus_{i=1}^{n} M_{n_{i}}\left(D_{i}\right)$. Since $\bar{A}$ has characteristic zero, so has one of the division rings $D_{i}$. Since $M_{n_{i}}\left(D_{i}\right)$ is a homomorphic image 
of $A, M_{n_{i}}\left(D_{i}\right)[G]$ is a homomorphic image of $A[G]$ and is semilocal. Now $M_{n_{i}}\left(D_{i}\right)[G] \cong M_{n_{i}}\left(D_{i}\right) \otimes_{Q} Q[G]$. By [4, Lemma 2], $Q[G]$ is semilocal. Hence $G$ is finite.

\section{REFERENCES}

1. S. Amitsur, On the semi-simplicity of group algebras, Michigan Math. J. 6 (1959), 251-253. MR 21 \# 7256.

2. I. G. Connell, On the group ring, Canad. J. Math. 15 (1963), 650-685. MR 27 \#3666.

3. E. Formanek, A problem of Herstein on group rings, Canad. Math. Bull. 17 (1974), 201-202. MR 50 \# 13118.

4. A. Rosenberg and D. Zelinsky, Tensor products of semiprimary algebras, Duke Math. J. 24 (1957), 555-559. MR 19, 727.

Department of Mathematics, University of Chicago, Chicago, Illinois 60637

Department of Mathematics, University of Manitoba, Winnipeg, Manitoba, Canada R3T 2N2 (Current address of S. M. Woods)

Current address: (John Lawrence): Department of Pure Mathematics, University of Waterloo, Waterloo, Ontario, Canada 